\title{
Perfil do uso do tabaco em estudantes de medicina em uma universidade particular de Sergipe
}

\author{
Profile of tobacco use among medical students at a private university in Sergipe \\ Perfil del consumo de tabaco entre estudiantes de medicina en una universidad privada \\ en Sergipe
}

Rebeca dos Santos Sirqueira ${ }^{1 *}$, Ana Celia Goes Melo Soares ${ }^{1}$, Mayana Lula Andrade ${ }^{1}$, Rafael Ribeiro Almico Fraga ${ }^{1}$, Thiago Lima Santos ${ }^{1}$, Amanda Silveira de Carvalho Dantas ${ }^{1}$, Roberta Souza Santos ${ }^{1}$, Antonio Souza Lima Junior ${ }^{1}$.

\section{RESUMO}

Objetivo: Traçar o perfil geral de estudantes do curso de medicina de universidade particular do estado de Sergipe que fazem uso do tabaco, bem como analisar seus padrões de uso e abuso. Métodos: Trata-se de um estudo descritivo e de abordagem quali-quantitativa. A amostra obteve 281 estudantes de medicina do $1^{\circ}$ ao $6^{\circ}$ ano. O instrumento de coleta foi um questionário próprio que abordava início de uso do tabaco, frequência, dependência, causas e consequências. Os dados foram analisados através de estatística descritiva. Resultados: Foi possível constatar que: $32 \%$ dos estudantes questionados fazem ou fizeram, em algum momento, uso do tabaco. Do universo total, $46,7 \%$ eram homens que faziam uso em contraste com $24,7 \%$ de mulheres. No que diz respeito a etapa do curso em que fizeram uso, as porcentagens maiores correspondem a $3^{\circ}(14,4 \%)$ e a $8^{\circ}(17,8 \%)$. Dos consumidores da droga, $52,4 \%$ nunca tentaram parar o uso e, $90 \%$ negaram quaisquer disfuncionalidades. Conclusão: Concluiu-se ser elevada a prevalência do tabagismo dentre os alunos de medicina da universidade avaliada, apesar de terem conhecimento da possibilidade das disfuncionalidades, e que tal prevalência é iniciada por fatores como o objetivo de diversão e redução de estresse.

Palavras-chave: Produtos do tabaco, Estudantes, Inquéritos e questionários, Tabagismo.

\begin{abstract}
Objective: To outline the general profile of medical school students from a private university in the state of Sergipe who use tobacco, as well as to analyze their patterns of use and abuse. Methods: This is a descriptive study with a qualitative and quantitative approach. The sample obtained was 281 medical students from the 1 st to the 6th year. The collection instrument was a specific questionnaire that addressed the beginning of tobacco use, frequency, dependence, causes and consequences. The data were analyzed using descriptive statistics. Results: It was possible to verify that: $32 \%$ of the questioned students use or have used tobacco at some point. Of the total universe, $46.7 \%$ were men who used it in contrast to $24.7 \%$ women. Regarding the stage of the course in which they used it, the highest percentages correspond to 3 rd (14.4\%) and 8th (17.8\%). Of the drug users, $52.4 \%$ never tried to stop the use and $90 \%$ denied any dysfunctionality. Conclusion: It was concluded that the prevalence of smoking among medical students at the university evaluated was high, despite the awareness of the possibility of dysfunctionalities, and that such prevalence is initiated by factors such as the objective of fun and stress reduction.
\end{abstract}

Keywords: Tobacco products, Students, Surveys and questionnaires, Smoking.

${ }^{1}$ Universidade Tiradentes (UNIT), Aracaju - SE. *E-mail: rebecasirqueira95@gmail.com 
RESUMEN

Objetivo: Esbozar el perfil general de los estudiantes de medicina de una universidad privada en el estado de Sergipe que usan tabaco, así como analizar sus patrones de uso y abuso. Métodos: Estudio descriptivo con enfoque cualitativo y cuantitativo. La muestra obtuvo 281 estudiantes de medicina del primer al sexto año. El instrumento de recolección fue un cuestionario específico que abordó el comienzo del consumo de tabaco, la frecuencia, la dependencia, las causas y las consecuencias. Los datos se analizaron mediante estadística descriptiva. Resultados: Fue posible verificar que: $32 \%$ de los estudiantes encuestados usan o han usado tabaco en algún momento. Del universo total, $46.7 \%$ fueron hombres que lo usaron en contraste con $24.7 \%$ mujeres. En cuanto a la etapa del curso en que lo utilizaron, los porcentajes más altos corresponden al $3{ }^{\circ}$ $(14,4 \%)$ y $8^{\circ}(17,8 \%)$. De los consumidores de drogas, el $52.4 \%$ nunca intentó dejar de usarlo, y el $90 \%$ negó cualquier disfuncionalidad. Conclusión: Se concluyó que la prevalencia del tabaquismo entre los estudiantes de medicina de la universidad evaluada era alta, a pesar de ser conscientes de la posibilidad de disfuncionalidades, y que dicha prevalencia se inicia por factores como el objetivo de la diversión y la reducción del estrés.

Palabras clave: Productos de tabaco, Estudiantes, Encuestas y cuestionarios, Fumar.

\section{INTRODUÇÃO}

O tabaco é uma erva - da qual é extraída a nicotina - que pode ser utilizado de diversas formas: mascado, aspirado, absorvido pela mucosa oral e, principalmente, fumado. Por muito tempo seu uso foi visto como uma opção de vida. É sabido e amplamente divulgado até mesmo pelos meios de comunicação de massa que o tabagismo é uma doença gerada pela dependência à nicotina que traz consigo diversos prejuízos à saúde e é considerada um dos maiores desafios da saúde pública no mundo atual (INCA/MS, 2020; BRASIL, 2004; RICHMOND R, 1999).

A fumaça vinda do tabaco é composta por uma mistura de gases e partículas com mais de 4.700 substâncias que são consideradas tóxicas pela capacidade de alterar o DNA das células, podendo, inclusive, culminar em câncer. Dentre as substancias estão o formaldeído, alcatrão, nitrosaminas e a própria nicotina. Esta última, é responsável pela dependência química e, uma vez absorvida, interage com receptores presentes na periferia dos neurônios que liberam uma série de substâncias químicas. Uma dessas substâncias, a dopamina, conduz o indivíduo à uma forte sensação de prazer e euforia, que fazem com que urja a necessidade de continuar fumando. Devido a tais reações químicas é que se pode concluir que o fumante não fuma porque quer, mas sim, porque precisa repor nicotina (AMS, 2013).

Segundo dados da Associação Médica Brasileira (MAS), anualmente cerca de 7 milhões de mortes são causadas pelo tabagismo ao redor do mundo que resultam em um custo para os governos de 1,4 trilhões de dólares. Fazendo um recorte no cenário brasileiro, 428 pessoas morrem por dia devido ao consumo do tabaco, resultando em uma perda de 56,9 bilhões de reais todos os anos por despesas médicas e perda de produtividade. Outrossim, 156.216 mortes anuais poderiam ser evitadas (AMS, 2013). O tabagismo é, portanto, um fator de risco evitável para a maioria das doenças cardiovasculares, - como hipertensão arterial e acidentes vasculares - pulmonares, - como, asma e doença pulmonar obstrutiva crônica - oculares, - como catarata - e oncológicas, com ênfase na leucemia mieloide aguda, no câncer de bexiga, câncer de pâncreas, câncer de fígado, câncer do colo do útero, câncer de esôfago, entre outros (INCA/MS, 2020; BUZATTO SV, SOLER ZASG, 2010).

Segundo a Organização Mundial de Saúde (OMS), o fumante pode ser classificado em fumante diário ou habitual e fumante ocasional. Há ainda a classificação do não fumante: ex-fumante, ex-fumante ocasional e não fumante propriamente dito (WHO, 1983).

Os profissionais da saúde desempenham um importante papel no que diz respeito ao aconselhamento ao abandono do fumo durante suas consultas ou através de campanhas contra o tabaco. Contudo, ainda que médicos reconheçam a morbimortalidade relacionada ao fumo, em geral orientam pouco seus pacientes para 
o abandono do tabagismo, possivelmente por não confiarem em sua própria habilidade e conhecimentos sobre técnicas que tenham este objetivo (MARTIN EC, et al., 2013). De acordo com Nerín I, et al. (2004) a educação médica não é tão aproveitada como deveria. Há uma falha no que diz respeito a educar a população a não começar a fumar e à cessação do tabagismo.

Nesse contexto, os graduandos de medicina também devem ser instruídos com o fim de, futuramente, servirem como modelo comportamental para população em geral. Há de se observar que a prevalência atual de tabagismo entre os estudantes varia de 10 a $20 \%$, com tendência a queda. No entanto, estudos mostram uma velocidade menor nos últimos anos em relação a que deveria ser esperada (MENEZES AMP, et al., 2001; MENEZES AMP, et al., 2004).

O dado acima pode ser justificado por se tratar o ingresso do jovem na vida acadêmica, um período acompanhado de intensa vulnerabilidade considerando ser uma etapa de descobertas, com mudança de ambiente e costumes, conquista de autonomia, descobrimento de si mesmo, instabilidade em relação a opiniões, necessidade de aprovação dos colegas, maiores cobranças e aumento das responsabilidades, além do afastamento do seio familiar (SILVA KG, et al., 2018).

Assim, diversas são as causas que podem levar o estudante ao uso do tabaco, entre elas a busca pela redução da ansiedade, a influência de amigos, e até mesmo vontade própria ou curiosidade (STRAMARI LM, et al., 2009).

Diante do contexto alarmante posto, foi detectada relativa escassez de trabalhos de rigor científico que descrevam a prevalência e o perfil de tabagistas especificamente entre os acadêmicos de medicina. Deste modo, o presente artigo concentra sua relevância na medida em que pretende servir a comunidade acadêmica subsidiando conhecimento para publicações outras, seguindo o objetivo principal de traçar o perfil geral de estudantes de medicina que fazem uso do tabaco, além de analisar seus padrões de uso e abuso.

\section{MÉTODOS}

Trata-se de um recorte de um estudo clínico transversal, descritivo e de abordagem analítica qualiquantitativa. $O$ universo dessa pesquisa foram todos os estudantes de Medicina de uma universidade particular (Universidade Tiradentes) do município de Aracaju, Estado de Sergipe. A amostra populacional almejada foi de 254 alunos, e teve seu cálculo baseado na fórmula de Gil (GIL, 2008), para populações finitas, que analisa o tamanho necessário desta.

Essa amostra foi baseada para uma população finita de 812 alunos no total matriculados dos doze semestres da graduação de medicina, totalizando os 6 anos do curso, na prevalência extrapolada de $40 \%$ do consumo de drogas lícitas. No entanto, foi possível analisar as respostas de um total de 281 alunos que efetivamente responderam ao questionário aplicado. O erro amostral obteve uma margem de $5 \%$ e uma confiança de $95 \%$.

Os critérios de inclusão foram estudantes acima de 18 anos de ambos os sexos, regularmente matriculados na etapa cursada e que assinaram o termo de consentimento livre e esclarecido (TCLE), de acordo com as normas do Conselho Nacional de Saúde do Ministério da Saúde explicitadas na resolução 466/12. O trabalho obteve aprovação do CEP, com o parecer CAAE 12088919.4.0000.5371.

Os dados foram coletados no campus Farolândia da Universidade Tiradentes em Aracaju-SE. O instrumento utilizado para essa coleta foi um questionário específico com informações sobre os tipos e a frequência de substâncias lícitas consumidas pelos estudantes; relação do consumo com o rendimento acadêmico, qualidade do sono e possíveis transtornos psiquiátricos associados.

Após a aplicação do questionário, os pesquisadores contabilizaram os dados alimentando-os em uma planilha eletrônica com os resultados acerca das respostas de cada participante. As variáveis categóricas foram descritas por meio de frequência absoluta e relativa percentual.

As associações entre déficit cognitivo e as demais variáveis categóricas foi testada por meio do teste QuiQuadrado de Pearson. As variáveis contínuas foram descritas por meio de média e desvio padrão. A 
aderência das variáveis contínuas à distribuição normal foi testada por meio do teste de Shapiro-Wilk. As diferenças nas medidas de tendência central foram testadas por meio do teste $T$ para amostras independentes (normal). O nível e significância adotado foi de 5\% e software utilizado foi o R Core Team 2020. Para efeito deste recorte os autores trabalharam na identificação do perfil do uso de tabaco em universitários de medicina desta amostra.

\section{RESULTADOS}

Foram avaliados 281 alunos de medicina de uma universidade particular do estado de Sergipe do $1^{\circ}$ ao $12^{\circ}$ período, ou seja, alunos de todas as etapas. Do total de alunos pesquisados, $32 \%$ relataram já ter feito uso de derivados do tabaco. Do universo de mulheres entrevistadas, apenas $24,7 \%$ faziam uso da droga, enquanto que no universo masculino $46,7 \%$ eram consumidores, ou seja, proporcionalmente mais homens fumam do que mulheres.

Em relação ao período atual dos acadêmicos que em algum momento já experimentaram a droga, é possível constatar, de acordo com a tabela 1, que o $1^{\circ}$ período e o $12^{\circ}$ possuem a mesma porcentagem de $7,8 \%$, e os maiores valores são o do $3^{\circ}$ período, com $14,4 \%$ e o do $8^{\circ}$ período com $17,8 \%$; ambas são etapas anteriores ao internato que se inicia no $9^{\circ}$ período, momento em que os alunos mais tem afinidade com procedimentos médicos, hospitais, professores, bem como em que o conhecimento prático se sobrepõe ao conhecimento teórico.

Tabela 1 - Prevalência do uso do tabaco de acordo com o período de estudantes de medicina.

\begin{tabular}{cc}
\hline Período & Frequência (\%) \\
\hline $1^{\circ}$ & 7,8 \\
$2^{\circ}$ & 4,4 \\
$3^{\circ}$ & 14,4 \\
$4^{\circ}$ & 3,3 \\
$5^{\circ}$ & 7,8 \\
6 & 11,1 \\
$7^{\circ}$ & 3,3 \\
$8^{\circ}$ & 17,8 \\
$9^{\circ}$ & 11,1 \\
$10^{\circ}$ & 2,2 \\
$11^{\circ}$ & 8,9 \\
$12^{\circ}$ & 7,8
\end{tabular}

Fonte: Sirqueira RS, et al., 2019.

Na primeira coluna da Tabela 2 foi analisada a frequência de uso em relação aos últimos 3 meses. Podese notar que $41,1 \%$ são não fumantes propriamente dito, $56,7 \%$ são fumantes ocasionais e $2,2 \%$ são fumantes diários. Considerando que são alunos que estudam doenças em que a maioria tem como fator de risco o tabaco, estes dois últimos valores são elevados.

Já na segunda coluna da mesma tabela, foi questionado se havia um forte desejo ou urgência em consumir tal substância nos últimos 3 meses. É interessante atentar para o fato de que pouco menos de $10 \%$ dos alunos entrevistados tiveram esse desejo semanalmente ou quase todos os dias. 
Tabela 2 - Frequência de uso do tabaco e de forte desejo de consumo do mesmo, nos últimos 3 meses.

\begin{tabular}{ccc}
\hline Frequência & Uso (\%) & Forte desejo (\%) \\
\hline Nenhuma & 41,1 & 72,2 \\
1 ou 2 vezes & 45,6 & 14,4 \\
Mensalmente & 3,3 & 5,6 \\
Semanalmente & 7,8 & 3,3 \\
Diariamente ou quase todos os dias & 2,2 & 4,4 \\
\hline
\end{tabular}

Fonte: Sirqueira RS, et al., 2019.

Foram feitas as mesmas duas perguntas anteriores acrescentando a concomitância com a ingestão de bebida alcoólica. 7,8\% dos que fizeram uso do tabaco nos últimos três meses não ingeriram bebida alcoólica em nenhum momento, $18,9 \%$ ingeriram 1 ou 2 vezes, $23,3 \%$ mensalmente, $48,9 \%$ semanalmente e $1,1 \%$ diariamente ou quase todos os dias. Já em relação ao forte desejo ou urgência de consumo, $40 \%$ das pessoas que faziam uso do tabaco não apresentaram vontade de beber bebidas alcoólicas em nenhum momento, $21,1 \% 1$ ou 2 vezes, $5,6 \%$ mensalmente, $27,8 \%$ semanalmente e $5,6 \%$ diariamente ou quase todos os dias.

Tais perguntas, tanto para o tabaco quanto para o álcool, foram feitas se baseando no questionário Cut down, Annoyed by criticism, Guilty e Eye-opener (CAGE) que identifica transtornos ou dependência associados ao uso do álcool.

O CAGE consiste em perguntas sobre a tentativa de diminuir ou cortar o álcool, se a pessoa já ficou incomodada ou irritada com outros porque criticaram seu jeito de beber, se já se sentiu culpada e se já teve que beber para aliviar os nervos ou reduzir os efeitos de uma ressaca. Na tabela 3, é possível verificar os motivos de uso. Se faz necessário frisar que os maiores valores correspondem aos motivos diversão e redução de estresse.

Tabela 3 - Motivos para o uso do tabaco.

\begin{tabular}{cc}
\hline Motivos & $(\%)$ \\
\hline Diversão & 67,8 \\
Redução de estresse & 20 \\
Socialização & 18,9 \\
Necessidade de melhora do desempenho acadêmico & 0 \\
Nenhuma das opções citadas & 4,4 \\
\hline
\end{tabular}

Fonte: Sirqueira RS, et al., 2019.

O uso do tabaco pode trazer diversas disfuncionalidades. Foi questionado se durante os últimos 3 meses houve como resultado problema de saúde, social, legal ou financeiro. $90 \%$ negaram qualquer tipo de problema, $5,6 \% 1$ ou 2 vezes, 2,2\% mensalmente, 1,1\% semanalmente, mesmo valor para diariamente ou quase todos os dias.

Outra pergunta do questionário inspirada no CAGE, foi a de tentativa de diminuir ou parar o uso do tabaco. $52,4 \%$ nunca tentaram, 3,3\% tentaram e não conseguiram, 3,3\% tentaram e logo depois retornaram com o uso, $37,8 \%$ tentaram e conseguiram.

Dos alunos que fizeram uso do tabaco e em algum momento já fizeram uso de substâncias com prescrição médica, apresentou relevância o uso de anfetaminas para manutenção de vigília (atomoxetina, lisdexanfetamina, modafilina, metilfenidato...) com $21,1 \%$ e o uso de hipnóticos/sedativos (remédios para dormir, ansiolíticos, tranquilizantes, barbitúricos, fenobarbital, pentobarbital, benzodiazepínicos...) com 54,4\%. Apesar de não haver dentro da bibliografia consultada estudos que corroborem ou discordem das taxas encontradas, diante de tal dado é possível que os alunos que fazem uso do tabaco tenham acompanhamento médico provavelmente psiquiátrico, ou consigam prescrições médicas através de contatos. 


\section{DISCUSSÃO}

De acordo com estudos de Werneck FA, et al. (2016), de 199 estudantes de medicina da Universidade Severino Sombra, $17 \%$ afirmaram já ter feito uso de tabaco. Houve uma prevalência de $14 \%$ de tabagismo entre os alunos da Universidade Federal Fluminense (JUNIOR CTS, et al.,2006), de 17,1\% com estudantes de medicina da federal de Sorocaba, (ROSEMBERG J, et al.,1990) na Universidade Federal de Pelotas de 10,1\% (MENEZES AMP, 2004) e 9,4\% em uma Universidade de Brasília em 2006 (ANDRADE APA, et al.,2006).

No estudo conduzido por Antunes JML, et al. (2017), em um universo de 325 pessoas, $36,9 \%$ afirmou ter feito uso. Somente nesta última análise, a porcentagem foi maior do que a averiguada no estudo em questão (com $32 \%$ ) ou seja, em um comparativo com os demais estudos, a porcentagem encontrada representa aproximadamente o dobro.

Em relação ao sexo, de acordo com Petroianu A, et al. (2010) 23,1\% dos homens consomem tabaco, em contraste com $9,9 \%$ das mulheres, cenário esse que corresponde àquele encontrado na amostra do presente estudo, e que permite a fácil conclusão de que os homens apresentam prevalência em relação as mulheres.

Foi verificado em estudos que no período do internato, correspondente ao quinto e sexto ano, faz-se menos uso do tabaco, com $16,8 \%$ e $14,9 \%$ dos estudantes, respectivamente, sendo que do primeiro ao quarto ano tal dado varia de 16,8 a 17,4\%, o que corrobora com nossa análise (STRAMARI LM, et al., 2019). Em contrapartida, no estudo de Magliari RT, et al. (2008), observou-se uma maior prevalência de tabagismo no quinto ano da amostra analisada com $32 \%$ dos tabagistas.

Este alto valor foi apresentado tendo como possíveis causas influência sofrida pelos amigos e uma considerável parcela dos estudantes mais velhos morando fora de casa. A média de tabagismo dos outros anos variou de $9,6 \%$ a $16 \%$. Outro estudo em espanhol, de Mas A, et al. (2004) está de acordo que a prevalência do tabagismo aumenta com o passar dos anos de faculdade.

No que diz respeito às classificações de fumante, em estudo realizado por Werneck FA, et al. (2016) na Universidade Severino Sombra, $8,5 \%$ se consideraram fumantes diários ou habituais e $8,5 \%$ fumantes ocasionais, $53,7 \%$ declararam nunca terem usado tabaco. No presente estudo, notou-se que há um número exorbitantemente maior de fumantes ocasionais, com $56,7 \%$ em contraste com um número muito menor de fumantes diários (2,2\%). $41,1 \%$ dos questionados nunca fumaram.

Ainda de acordo com o estudo de autoria de Werneck FA, et. al. (2016) sobre a necessidade de fumar juntamente com o consumo de bebida alcoólica, 61,62\% não sentem necessidade, 10,46\% sentem necessidade sempre e $26,73 \%$ sentem uma vez ou outra. Não houve discrepância significativa quando comparado aos estudantes consultados da pesquisa, tendo em vista que $40 \%$ não apresentaram desejo ou urgência em fazer uso do tabaco e o álcool juntos, 33,4\% apresentaram semanalmente ou quase diariamente.

Entretanto, quando questionado sobre a frequência do uso concomitante nos últimos três meses, 7,8\% não fizeram uso, e $50 \%$ fizeram uso dos dois juntos. Tal dado demonstra que há diferença entre os que sentem necessidade/urgência e os que realmente chegam a fazer uso, estes últimos com taxa elevada. A informação permite ainda interpretar que há uma forte relação entre álcool e tabaco. Ainda em concordância, em uma pesquisa realizada por Almeida JB, et al. (2011) com universitários de uma universidade do interior de São Paulo 74,4\% associam o uso do tabaco à ingestão de álcool.

No tangente à disfuncionalidade causada pelo uso do tabaco houve estudo em que $8,3 \%$ de fumantes em universidade local reportaram que a vontade/necessidade de fumar teve influência negativa sobre 0 desempenho e/ou concentração relacionados às atividades acadêmicas nos últimos 12 meses. Fato esse que se relaciona com o nosso estudo, em que $10 \%$ relataram ter tido algum problema de saúde, social, legal ou financeiro.

Não havia trabalhos onde se pudesse relacionar o uso do tabaco com problemas legais ou financeiros. É interessante atentar para o fato de que de acordo com Coelho AC, et al. (2010) a maioria dos estudantes sabe que o tabagismo é prejudicial à saúde. Entretanto, pouco menos da metade dos alunos pensava que o 
tabagismo era a principal causa evitável de morte no mundo. Melani, et al. (2000) avaliaram os conhecimentos de estudantes de medicina do primeiro ano do curso de medicina e $92 \%$ responderam que o tabagismo era prejudicial à saúde. Ou seja, é possível inferir que os alunos têm uma baixa porcentagem no que diz respeito a disfuncionalidade agora, mas tem conhecimento da possibilidade de vir a ter.

Em relação aos motivos que levaram os pesquisados a começar a fumar, na presente pesquisa foram relatados: diversão $(67,8 \%)$, redução de estresse $(20 \%)$ e socialização $(18,9 \%)$. Em uma Universidade de Passo Fundo, verificou-se que $69,2 \%$ dos fumantes iniciaram o tabagismo entre 15 e 19 anos, tendo como principais motivações a vontade própria e/ou a influência dos amigos. Não há muitos estudos relacionados especificamente a estudantes de medicina e suas causas de início. Foi apresentado de forma geral entre estudantes de Brasília que a vontade própria foi o mais citado, com $57,6 \%$ das respostas. Em seguida, $23,2 \%$ citaram a influência dos amigos, 8,5\% o modismo e 4\% a influência dos pais (ANDRADE APA, et al., 2016; STRAMARI LM, et al., 2009). São necessários novos estudos em relação a causa, visto que é uma forma de prevenção ao uso do tabaco. Discutir e trabalhar com as possíveis causas de início do tabagismo leva a diminuição do seu uso e consequentemente, do seu abuso.

No nosso estudo, no tocante à tentativa de diminuir ou parar o uso do tabaco, constatou-se $52,4 \%$ nunca tentaram, 3,3\% tentaram e não conseguiram, 3,3\% tentaram e logo depois retornaram com o uso, 37,8\% tentaram e conseguiram. Já na Universidade de Passo Fundo, dos fumantes, 67,3\% já haviam tentado parar de fumar, $96,0 \%$ acreditavam serem capazes de parar de fumar e $87,2 \%$ pretendiam deixar de fumar. Diante desse cenário, é possível observar que ainda que seja um número elevado, mais da metade dos que fumam, tentaram parar (STRAMARI LM, et al., 2009). Talvez, com um maior incentivo promovido através dos agentes responsáveis por políticas de saúde, os fumantes tenham êxito na trajetória que conduz à erradicação do habito em questão.

\section{CONCLUSÃO}

Conclui-se por meio dos resultados estatísticos analisados no artigo que há uma prevalência elevada do uso do tabaco em estudantes de medicina em uma universidade particular de Sergipe quando comparada com outras, e destes, mais da metade nunca tentou parar. Em resposta ao objetivo da presente pesquisa foi possível traçar o perfil geral do uso do tabaco que corresponde em sua maioria a homens, fumantes ocasionais e que possuem conhecimento da possibilidade de disfuncionalidades futuras, ainda que não as tenham no presente. Além disso, a análise dos padrões de uso e abuso nos conduziu a resposta de que há, de fato, forte relação entre o álcool e o tabaco, que o uso e/ou abuso das substâncias podem ocasionar problemas de saúde, sociais e/ou financeiros, e dentre os motivos que estimulam o início do uso do tabaco estão, principalmente citados, a busca pela diversão, redução de estresse e socialização. Diante deste cenário, são necessárias maiores medidas ou projetos no que diz respeito a cessação do uso do tabaco, bem como a monitoração da relação que pode estar estabelecida entre o curso de medicina e suas causas de início.

\section{AGRADECIMENTOS}

Agradecimento aos estudantes de medicina de uma universidade particular de Sergipe por incentivar a pesquisa científica colaborando com sua realização.

\section{REFERÊNCIAS}

1. ALMEIDA JP, et al. Prevalência e características do tabagismo na população universitária da região de Lins-SP. Rev Bras Enferm, Brasília 2011 mai-jun; 64(3): 545-50.

2. ANDRADE APA, et al. Prevalência e características do tabagismo em jovens da Universidade de Brasília. J Bras Pneumol. 2006;32(1):23-8.

3. ANTUNES JML, BORTOLI S. Perfil do uso de drogas lícitas e ilícitas entre os alunos do ensino superior da universidade estadual de ponta grossa. UEPG Ci. Biol. Saúde, 2017; v.23, n.2, p. 134-143. 
4. ASSOCIAÇÃO MEDICA BRASILEIRA (AMS) et, al. Evidências Científicas sobre Tabagismo para Subsídio ao Poder Judiciário. 2013. 71 p. Disponível em: https://diretrizes.amb.org.br/_BibliotecaAntiga/tabagismo-para-subsidio-aopoder-judiciario.pdf. Acesso em 28 fev. 2020.

5. BUZATTO SV, SOLER ZASG. Tabagismo e etilismo entre acadêmicos de medicina e enfermagem da Faculdade de Medicina de São José do Rio Preto - SP - FAMERP. Arq Ciênc Saúde, 2010; 17(3):122-7.

6. BRASIL. Ministério da Saúde. Instituto Nacional do Câncer. Por um mundo sem tabaco: mobilização da sociedade civil. Ação global para o controle do tabaco o 1ํTratado Internacional de Saúde Pública. 3a ed. Rio de Janeiro: INCA, 2004.

7. COELHO AC, et al. Conhecimentos sobre tabagismo entre estudantes de medicina. Rev HCPA. 2010;30(2):94-99.

8. FAGERSTRÖM K. Uma breve apresentação da neurofarmacologia e fisiologia da dependência à nicotina. In: Gigliotti AP, Presman S, eds. Atualização no tratamento do tabagismo. Rio de Janeiro: ABP Saúde; 2006.

9. GIL AC. Métodos e técnicas de pesquisa social. São Paulo: Atlas; 2008.

10. INSTITUTO NACIONAL DE CÂNCER (INCA/MS). Tabagismo. In causas e prevenção. 2020. Disponível em: https://www.inca.gov.br/tabagismo\#_edn4. Acesso em: 22 fev. 2020.

11. JUNIOR CTS, et al. Prevalência de tabagismo entre estudantes de graduação em medicina da Universidade Federal Fluminense. Pulmão RJ 2006;15(1):11-15.

12. KIRCHENCHTEIN C, CHATKIN JM. Dependência da nicotina. In: Diretrizes para cessação do tabagismo. J Bras Pneumol. $2006 ; 30$ (suppl. 2):11-8.

13. LONDON ED, et al. Distribution of cerebral metabolic effects of nicotine in the rat. Eur J Pharmacol. 1985;110:391-2.

14. MAGLIARI RT, et al. Prevalência de tabagismo em estudantes de faculdade de medicina. Rev Med (São Paulo). 2008; 87(4):264-71.

15. MARTIN EC, et al. O tabagismo e a formação médica. Rev Bras Educ Med. 2003;27(3):177-83.

16. MAS A, et al. Smoking habits among sixthyear medical students in Spain. Arch Bronconeumol. 2004; 40(9):403-8.

17. MELANI AS et, al. Tobacco smoking habits, attitudes and beliefs among nurse and medical students in Tuscany. Eur J Epidemiol. 2000;16(7):607-11.

18. MENEZES AMP, et al. Evolução temporal do tabagismo em estudantes de medicina, 1986, 1991, 1996. Ver Saúde pública. 2001;35:165-9.

19. MENEZES AMP, et al. Tabagismo em estudantes de Medicina: tendências temporais e fatores associados. J. Bras. Pneumologia; 30(3):223-8, 2004.

20. NERÍN I, et al. Evalua-tion of the influence of medical education on the smoking attitudes of the future doctors. Arch Bronconeumol. 2004;40(8):341-7.

21. PETROIANU A, et al. Prevalência do consumo de álcool, tabaco e entorpecentes por estudantes de medicina da universidade federal de minas gerais. Rev Assoc Med Bras, 2010; 56(5): 568-71.

22. POMERLEAU CS, POMERLEAU OF. Euphoriant effects of nicotine in smokers. Psychopharmacology. 1992;108(4):460-5.

23. PONTIERI FE, et al. Effects of nicotine on the nucleus accumbens and similarity to the addictive drug. Nature 382 , 1996; 255-257.

24. RICHMOND R. Teaching medical students about tobacco. Thorax 1999;54:70-8.

25. RONDINA RG, et al. A relação entre tabagismo e características socio-demográficas em universitários. Psicologia, Saúde \& Doenças, 2005, 6 (1), 35-45.

26. ROSEMBERG J, PERIN S. Tabagismo entre estudantes da Faculdade de Ciências Médicas de Sorocaba. Tabagismo nos acadêmicos de medicina e nos médicos. J Pneumol 1990;16:13-22.

27. SILVA KG, et al. Análise do consumo de estimulantes cerebrais por estudantes universitários: Uma revisão de literatura. Revista Interdisciplinar de Ciências Médicas - Teresina-PI. 2018. ISSN 2594-522X.

28. STRAMARI LM, et al. Prevalência e fatores associados ao tabagismo em estudantes de medicina de uma universidade em Passo Fundo (RS). J Bras Pneumol. 2009;35(5):442-448.

29. SHERWOOD N. Effects of nicotine on human psychomotor performance. Human Psychopharmacol. 1993;8(3):15584.

30. WERNECK FA, et al. Prevalência do tabagismo entre os estudantes de Medicina da Universidade Severino Sombra. Revista de Saúde. 2016; 07 (2): 08-11.

31. WHO. Smoking control Strategies in Developing countries. WHO Expert Committee, Geneva, 1983. (Techmical Report Series 695). 